\title{
In einem Boot
}

\section{Eine Fachtagung untersuchte die Schnittstellen von Jugendhilfe und Schule}

KERSTIN LANDUA

Kerstin Landua ist Leiterin der Arbeitsgruppe der Fachtagungen Jugendhilfe im Deutschen Institut für Urbanistik.

landua@difu.de

\author{
Beide oft konkurrierenden Systeme Jugendhilfe und \\ Schule haben ihre je eigenen Stärken und Schwächen \\ im Vergleich zum jeweils anderen System. Es gilt, \\ diese Besonderheiten mit dem Ziel der Förderung und \\ Motivation von Kindern und Jugendlichen zu nutzen.
}

Die Fachtagung »Schulversäumnisse Jugendhilfe und Schule in einem Boot? « wurde am 14. und 15. März 2013 in Potsdam von der Arbeitsgruppe Fachtagungen Jugendhilfe im Deutschen Institut für Urbanistik veranstaltet. Veranstaltungsort war das Tagungszentrum der Hoffbauer gGmbH auf der Insel Hermannswerder, weil dort das Jugendhaus OASE als freier Träger der Jugendhilfe seine Heimat hat und ein erfahrener Partner ist, was »Schulversäumnisse « angeht.

\section{Hikikomori - was Japan mit Schulversäumnissen in Deutschland zu tun hat}

Bodo Ströber, Leiter des Jugendhauses OASE in Potsdam, fragte zu Beginn seines Vortrages, ob Schulversäumnisse immer auch gleichzusetzen seien mit Schulverweigerung. Und wer definiere eigentlich, was »draußen « und was »drin« ist?

Es war ein sehr emotionaler Vortrag, der zum Nachdenken anregen und Anlass sein sollte, eigene Haltungen $\mathrm{zu}$ überprüfen. Er erzählte von einer Bildungsreise nach Japan und einem Phänomen, das er dort kennengelernt und das ihn nachhaltig beeindruckt hat: »Hikikomori«. Dieses Phänomen beschreibt (junge) Menschen, die einfach verschwinden, nicht nur aus der Schule, sondern aus jedem sozialen Leben überhaupt, aus der Arbeit, aus der Familie, aus den Strukturen des Alltags und letztendlich damit auch aus der Gesellschaft. Eine Art Generalverweigerung und Selbstaufgabe.
Erstaunlich war, dass die Frage nach dem Warum in Japan nicht thematisiert wird, sondern "man « von den deutschen Fachkolleginnen und Fachkollegen wissen wollte: Wie werden wir das Problem wieder los? Die Frage nach dem Warum bewegte aber die deutschen Experten sehr. Es gebe kaum zwei Fälle von Schulversäumnissen in seiner bisherigen Praxis im Jugendhaus OASE, die gleich seien. Jeder Fall sei ein Einzelfall. Es müsse immer überlegt werden, welche individuellen Probleme, aber auch Ressourcen bei den Jugendlichen vorhanden seien.

Die Frage, in welcher Form Jugendhilfe für das Kind hilfreich sein kann, stehe im Mittelpunkt. Der Wert pädagogischen Handelns bestehe darin, sich in jedem Einzelfall individuell und unvoreingenommen den jeweiligen Situationen der Jugendlichen zu widmen und daran das Handeln auszurichten. Die pädagogische Kurzinterpretation seiner Arbeit heißt: Störungen haben Vorrang.

Schon bei den Reformpädagogen könne man nachlesen, dass Lernen ein Akt der Befreiung sein sollte. Ein Problem dabei sei die Art, wie "wir« bilden. Eine Verbindung von Lernen und Spaß müsse da sein. Wichtigste Bedingung für die Förderung des Lernens sei die Qualität des Schüler-Lehrer-Verhältnisses. Beziehung ist die Grundlage allen pädagogischen Handelns und es dürfe dann nicht heißen: »Du bist hier in Mathe, wenn Du ein Problem hast, geh' zum Schulsozialarbeiter. « Es werde immer gefragt: Ist das Kind bereit für die Schule? - und nicht, ob die Schule auch bereit für das Kind ist. 


\section{"Wir haben ein Vertrauen zu verlieren«}

Über »Schulversäumnisse + Rechte der Kinder?«, also rechtliche Grundlagen, deren Möglichkeiten und Grenzen, den Umgang mit Datenschutz und Gefährdungseinschätzungen referierte Prof. Dr. Peter Knösel von der Fachhochschule Potsdam. Da es in Deutschland eine gesetzlich verankerte Schulpflicht gebe, müsse jedes Kind zur Schule gehen. Der juristische Bereich bei Schulversäumnissen reiche vom Bußgeld, vom unmittelbaren Zwang bis zu familienrechtlichen und strafrechtlichen Sanktionen.

Die Kooperationsmöglichkeiten mit den Eltern verschlechtern sich allerdings in der Regel, wenn juristische Mittel in Anspruch genommen werden. Beim Datenschutz müsse immer auf Zweck, Erforderlichkeit, Verhältnismäßigkeit und Transparenz geachtet werden: »Im Datenschutz ist alles verboten, außer ein Gesetz erlaubt es.«

Wenn fremde Systeme in die Kooperation geholt werden, brauche es die Erlaubnis der Eltern. »Hier haben wir ein Vertrauen zu verlieren.«In Bezug auf Kinderschutz sei das Schlüsselwort »Kindeswohlgefährdung « Eine Lehrerin beispielsweise könne als Privatperson alles fragen, was sie über ein Kind wissen möchte. Als Vertreterin der Behörde Schule bräuchte sie jedoch in

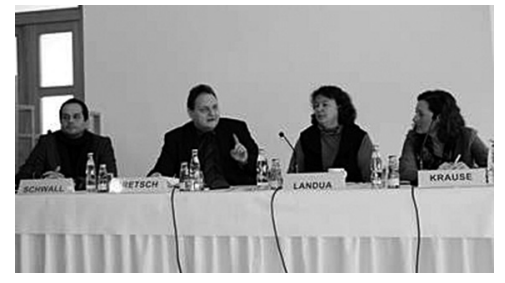

Die umfassende Dokumentation zu der Fachtagung "Schulversäumnisse - Jugendhilfe und Schule in einem Boot?" am 14. und 15. März 2014 in Potsdam ist beim Herausgeber und im Buchhandel erhältlich.

"Schulversäumnisse - Jugendhilfe und Schule in einem Boot?" Aktuelle Beiträge zur Kinder- und Jugendhilfe 89, Berlin 2013, 172 Seiten, 19,- Euro, ISBN-13: 978-3-931418-96-0

Deutsches Institut für Urbanistik gGmbH, Zimmerstraße 13-15, 10969 Berlin, Telefon o30 39001-0, E-Maildifu@difu.de, Internet www.fachtagungen-jugendhilfe.de der Regel die Einwilligung der Eltern. Für den Kinderschutz sei diese Schwelle aufgehoben.

Die Lehrerinnen und Lehrer müssen die Eltern im Gespräch über Kooperationsangebote aufklären und darauf hinweisen, dass es ihre Aufgabe als pädagogische Fachkräfte ist, das Wohl des Kindes zu schützen und das Jugendamt $\mathrm{zu}$ informieren, wenn die Eltern diese Angebote nicht wahrnehmen. Das sei die Rechtslage seit dem im Jahre 2012 in Kraft getretenen »Gesetz zur Kooperation und Information im Kinderschutz«.

\section{"Schulverweigerer mit Zukunft"}

Margret Schettler, Mitarbeiterin in der Landeskooperationsstelle Schule - Jugendhilfe, kobra.net $\mathrm{GmbH}$ Brandenburg, sprach über "Schulversäumnisse - Anzeichen und Ursachen «. Sie sagte zu Beginn ihres Vortrags: »Wir sind eine Truppe, die es nicht geben muss." Auftrag ihrer Arbeitsstelle sei es, die Zusammenarbeit von Schule und Jugendhilfe zu initiieren, zu unterstützen, zu begleiten, zu beraten und Prozesse zu moderieren.

In ihrem Arbeitskontext sei insbesondere die Frage wichtig: »Was passiert mit den Kindern, die zumeist hoffnungsfroh in die Schule kommen und später zu Schulverweigerern werden? « Hierzu ließ sie zunächst die Jugendlichen selbst zu Wort kommen und zeigte Filmausschnitte aus dem Dokumentarfilm "Schulverweigerer mit Zukunft", der im Jahr 2010 im Auftrag des Bildungsministeriums Brandenburg entstanden ist.

Die Referentin machte in ihrem Vortrag darauf aufmerksam, dass Schulversäumnisse in der Regel eine längere Vorgeschichte haben. Erfolgreiches Lernen brauche eine Befriedigung der (physischen und psychischen) Grundbedürfnisse, positive Vorerfahrungen, Motivation und Wohlbefinden. Dies sei jedoch nicht immer gegeben. Ursachen und Risikofaktoren für Schulversäumnisse seien sowohl bei den jungen Menschen selbst, in der Familie, in der Schule als auch bei der Gruppe der Gleichaltrigen zu suchen.

Beide Systeme, Jugendhilfe und Schule, hätten ihre Besonderheiten und Stärken im Vergleich zum jeweils anderen System, die es gelte mit dem Ziel der Förderung und Motivation der Kinder und Jugendlichen zu nutzen.
Anschließend fand ein Erfahrungsaustausch in sechs Arbeitsgruppen statt, in denen "Best Practice « vorgestellt und diskutiert wurde.

Der Input dieser Arbeitsgruppen wie auch alle Referate im Plenum sind in der Dokumentation zu dieser Tagung nachzulesen.

\section{Kooperation statt Grabenkämpfe}

Der zweite Veranstaltungstag begann mit einem Gespräch zum Bildungsauftrag von Schule. Gefragt wurde, was sich an Schule ändern muss, damit Kinder und Jugendliche (zurück) in die Schule gehen, was der Auftrag der Kinder- und Jugendhilfe bei Schulversäumnissen ist und welche Aufgabe die Jugendhilfe hier aus Sicht des Kindes hat. Gesprächspartner dabei waren Carolin Krause, Leiterin des Jugendamtes Köln, Frank Bretsch, Schulrektor der Ehm-Welk Oberschule Angermünde, sowie Stefan Schwall, Leiter des Instituts apeiros, Wuppertal.

Frank Bretsch sagte, das besondere Dilemma im Verhältnis zwischen Schule und Jugendhilfe bestehe in der vollkommen unterschiedlichen Definition der inhaltlichen Arbeit. Schule werde häufig von der Seite der Jugendämter und Freien Träger nicht nur kritisiert, sondern auch mit Veränderungswünschen überhäuft.

Allerdings habe Schule keineswegs die Aufgabe, alle sozialpädagogischen Aufträge und Herausforderungen durchzudiskutieren. Schule habe tatsächlich »knallharte« Aufgaben mit klaren Zeitvorgaben, die in ihrer Endgültigkeit von keiner sozialpädagogischen Arbeit erreicht werden müssen, weil in der Jugendarbeit der Weg das Ziel ist.

Wer Schule auf diesen Weg bringen wolle, handele verantwortungslos. Um Schülerinnen und Schüler nicht durch Schwänzen zu verlieren, sind viele Schulen bereits Wege gegangen, die einerseits öffentlich nur begrenzt wahrgenommen würden, andererseits auch noch viel mehr Nachahmer benötigten. Der Ehrlichkeit halber müsse gesagt werden, dass manche Kinder schon so kaputt in der Schule ankommen, dass die Arbeit mit ihnen im normalen Rahmen von Schule nicht möglich sei, weil sie eine völlig andere Umgebung und einen eingeschränkten Personenkreis ihres Vertrauens bräuchten. »Dieser Herausforderung stellen sich Schulen durch Schul- 
projekte, die es unter anderem ermöglichen, auf den einzelnen Jugendlichen bezogene Angebote zu unterbreiten und sich die Zeit zu nehmen, die wirkungsvollsten Bereiche herauszufinden - unter Veränderung der formalen und inhaltlichen Rahmenbedingungen. Dazu erklären sich Lehrer bereit, gemeinsam mit Sozialarbeitern tätig zu sein, ohne dass sie sich gegenseitig davon überzeugen wollen, dass der eine die Aufgabe besser löst als der andere.«

Für Stefan Schwall war es »wichtig zu betonen, dass man zwischen Bildung und Schule unterscheiden sollte. Das ist sinnvoll und das tun Sie vielleicht schon. Aus dieser Unterscheidung wird etwas Neues wachsen. Es gibt zahlreiche intelligente Lösungen in vielen Varianten, die die verschiedenen Institutionen zusammenbringen. Erfreulich sei, wie durch gelebte Kooperation versucht werde, institutionelle Schranken aufzulösen, und sicher spannend weiter $\mathrm{zu}$ verfolgen, ob das einen messbaren Effekt hervorbringe und Schule verändere.

Die Erkenntnis von Carolin Krause war, »dass wir das Problem nicht wieder loswerden, aber wir können daran arbeiten, dass es uns gemeinsam mehr Spaß macht, an dem Problem zu arbeiten «. Es sei wichtig, dafür zu sorgen, dass engagierte Menschen auch engagiert bleiben, dass Lehrer gern in die Schule gehen und »ihre« Kinder mögen. Nicht jeder Lehrer schaffe das sein gesamtes Berufsleben lang. "Wir können nur gemeinsam daran arbeiten, dass es allen mehr Spaß macht, mit diesem Problem zu leben, und es nicht zu groß werden zu lassen.«

\section{"Ich fühle mich so, wie ich bin, nicht anerkannt"}

Über »Schulversäumnisse und die $\mathrm{Zu}$ sammenarbeit mit der Kinder- und Jugendpsychiatrie « referierte Petra Vasen, Fachkraft für Kinder- und Jugendpsychiatrie von der Klinik für Psychiatrie und Psychotherapie des Kindes- und Jugendalters im LVR-Klinikum Essen. Eingangs sagte sie, viele Kinder und Jugendlichen würden sinngemäß mit dem Satz »Ich fühle mich so, wie ich bin, nicht anerkannt « in der Beratungsstelle ankommen.

Der Fokus des Vortrages von Petra Vasen lag auf Arbeitsweisen und Erfahrungen im Umgang mit Schulvermeidung im interdisziplinären Kontext und ganz konkret auf einer Vorstellung des Essener Schulvermeider-Projektes und des dazugehörigen Manuals. Sie nahm dabei Bezug auf die Aspekte motivationale Arbeit, kognitive Verhaltenstherapie, schulische Beratung, Sport-Coaching und Familienberatung.

Ausgangspunkt des Projektes war die Feststellung, dass die Schnittstelle zwischen Schule und Kinder- und Jugendpsychiatrie sowie zwischen Kinderund Jugendpsychiatrie und Kinder- und Jugendhilfe in Essen suboptimal war. Vor diesem Hintergrund wurde ein Therapie- und Beratungsnetzwerk gebildet, dem Essener Schulen sowie die aktiv oder passiv schulverweigernder Schülerinnen und Schüler innerhalb eines Jahres in das reguläre Schulsystem.

Elternarbeit geschehe hauptsächlich im Kontext von Hilfeplanung. Ein wichtiger Bestandteil ihrer Arbeit sei die Elternarbeit mit Familien mit Migrationshintergrund. Die Haltung dieser Eltern zu schulischen Problemen ihrer Kinder sei abhängig vom Grad der Integration in die deutsche Gesellschaft und Schul-, Bildungs- und Ausbildungssysteme seien oft fremd und für die Eltern nicht durchschaubar. Schwierig in der Zusammenarbeit mit den Eltern insgesamt sei u. a. eine gewisse Abwehrhal-

\section{"Seder Fall von Schulverweigerung ist ein Einzelfall mit je eigenen Konstellationen "}

Beratungsstelle in der Klinik angehören. Ratsuchende seien u. a. Eltern, der Allgemeine Soziale Dienst des Jugendamts und der Schulpsychologische Dienst.

In der Beratungsstelle findet ein erstes Screening mit den Schülerinnen und Schülern statt, wo Ursachen der Schulvermeidung liegen könnten. Hauptbestandteil im Essener Schulvermeiderprojekt sei die interdisziplinäre Zusammenarbeit. Dazu wurde ein multiprofessioneller Fachbeirat gegründet. Einigkeit habe darüber bestanden, dass eine schnelle Hilfe gewährleistet sein soll, da die Erfahrung lehre: Je länger ein Schüler abwesend ist, desto dauerhafter wird die Schulvermeidung und umso schlechter greifen die Interventionsmaßnahmen.

\section{Alle Eltern wollen gute Eltern sein - Elternarbeit}

Über präventive Ansätze in der Elternarbeit bei Schulversäumnissen in den Projekten »Die 2. Chance" und »Jugendmanufaktur « referierten Ulrike Behn, Diplom-Sozialpädagogin und therapeutische Fachkraft, sowie Ulrike Braiger, Diplom-Sozialpädagogin und Casemanagerin vom Schulprojekt KEEP sChOOL des Diakonischen Werks Tempelhof-Schöneberg e. V. in Berlin.

Zielgruppe der Projekte sind Schülerinnen und Schüler, die mindestens zwölf Jahre alt sind und sich maximal am Beginn der letzten Klasssenstufe befinden oder bereits berufsschulpflichtig sind. Angestrebt wird die Reintegration tung gegenüber Schule, aber dennoch werde ein intensiverer Kontakt zur Schule immer dann eingefordert, wenn es negative Tendenzen in der schulischen Entwicklung ihrer Kinder gibt.

Als hilfreich für die Reintegration der Kinder habe sich ein regelmäßiger Kontakt zwischen Eltern und Schule erwiesen sowie die Einrichtung eines runden Tisches mit den Eltern und den jungen Menschen, damit diese lernen, Verantwortung für sich selbst übernehmen und Lösungsvorschläge mit entwickeln können. Familie sei eine große Ressource und alle Eltern wollen gute Eltern sein. Dieses Potenzial müsse man nutzen, um nicht zuletzt Eltern auch dabei zu helfen, die Beziehungen zu ihrem Kind möglichst wieder in einen als positiv erlebten Kontext zu bringen.

\section{Ab auf die Insel}

Im Sinne eines offenen Ausklangs der Tagung bestand die Möglichkeit, an geführten Rundgängen über die Insel Hermannswerder teilzunehmen. Diese Rundgänge wurden von Jugendlichen aus dem Jugendhaus OASE vorbereitet und gestaltet, die sowohl über die Inselgeschichte als auch über die Arbeit des Jugendhauses OASE mit seinen vielfältigen Möglichkeiten zu erzählen wussten.

Das Angebot, gelebte Kooperation von Jugendhilfe und Schule vor Ort kennenzulernen und miteinander ins Gespräch zu kommen, nahmen viele der Tagungsteilnehmer gern an. 